\title{
Traveler program
}

\section{Fatema ahmed mohamed, Shimaa othman zaki ,Samaa mohamed abd el fatah, Hadeer khalifa abd el moez, Yasmeen ahhmed salah, Wessam H Elbehaidy}

\author{
Faculty of computers \$ Artificial intelligence, Helwan \\ university \\ Corresponding Email *: w_behaidy@fci.helwan.edu.edu
}

\section{INTRODUCTION}

Our idea is about capturing a photo for a landmark and retrieve some information about this landmark to help the tourist to know a lot about this place.

\subsection{Basic idea}

Perhaps the easiest way to obtain information about something is to use a picture of the object as a query. Consider, for instance, the tourist is in front of a monument and wants to have information about it. A very easy and intuitive action can be that of pointing the monument with a smartphone camera and obtaining pertinent and contextual information.

\subsection{Motivation}

With the technology growing in the mobile industry, it has become almost necessary for any business to be active on mobile and social media platforms and travel and tourism industry is not an exception. A huge part of the success of tourism and Travel has been played by Mobile Apps. As the partnership between the tourism industry and technology grows both have earned several grand slams. The days of guidebooks, maps, and other printed stuff have been replaced by Mobile Apps for Tourism and Travel Industry that come with functionalities. Need for Mobile App for Travel and Tourism. Now, the world is at the fingertips of mobile users. Mobile users are now getting real-time information which they need anytime, anywhere. More than 150 million travelers who have smartphones prefer apps for hotel room booking, and flight booking, etc. Among tour operators and tourists, Mobile App for Travel industry brings happiness with multi-benefits. and This application is important 
because it gives me more information about the tourist location and more information to enjoy the place Get the correct information about the place And the application of the mobile phone I can use it anywhere and anytime I do not need a tour guide. I would rather choose the language of my country as well as the least expensive for.

\section{Methodology:}

We were followed some steps in our implementation to this system that helped us get better results.

\subsection{Step1: Collecting dataset.}

We use a turkey dataset [link to dataset], this data consists of 200 classes. we faced some problems such as the number of image in each class is not equal (the difference between the number of image is huge), each class has a lot of image not related to the place (not belong to the name of class), also the data has no label, so we had to collect the data and add the label to each image.

this is not very efficient but since we had no more options, so we handled the situation under the circumstances.

in our system we work only on 6 classes, each class contain from 400 to 500 image.

\subsection{Step2: Preprocessing.}

Pre-Processing is the use of computer algorithms to perform image processing on digital images. It allows a much wider range of algorithms to be applied to the input data and can avoid problems such as the build-up of noise and signal distortion during processing.

The aim of it is an improvement of the image data that suppresses unwanted distortions or enhances some image features important for further processing.

Image Data Pre-Processing is much used for Neural Networks (Deep Learning) and has well-known steps are Uniform aspect ratio, Image Scaling, Mean, standard deviation of input data, Normalizing image inputs, Dimensionality reduction and Data augmentation.[1]In our system we preprocessing our dataset by resized all the image in dataset.

\subsection{Step3: Classification step.}

Image classification is the task of classifying a given image into one of the predefined categories.

Traditional pipeline for image classification involves two modules: feature extraction and classification. 
Feature extraction involves extracting a higher level of information from raw pixel values that can capture the distinction among the categories involved.

The problem is that feature extraction cannot be tweaked according to the classes and images.

So if the chosen feature lacks the representation required to distinguish the categories, the accuracy of the classification model suffers a lot, irrespective of the type of classification strategy employed, But with Convolutional Neural Networks(ConvNets), the task of training the whole network from scratch can be carried out using a large dataset like Imagenet. The reason behind this is, sharing of parameters between the neurons and sparse connections in convolutional layers.

in this step we try more than one model, we use Resnet, CNN, KNN, Vgg16.

We are trying more than one model to can perform better in our system and compare the results to work with the best and highest one given accuracy.

\section{3-System results and system snapshots}

\begin{tabular}{|l|l|}
\hline Model & Accuracy \\
\hline KNN & $82 \%$, with $\mathrm{k}=3$ \\
\hline KNN & $97 \%$, with $\mathrm{k}=2$ \\
\hline CNN & $88 \%$ \\
\hline VGG16 & $86.20 \%$ \\
\hline RESNET & $84 \%$ \\
\hline
\end{tabular}

[link to dataset] [2] 


\section{System snapshots}
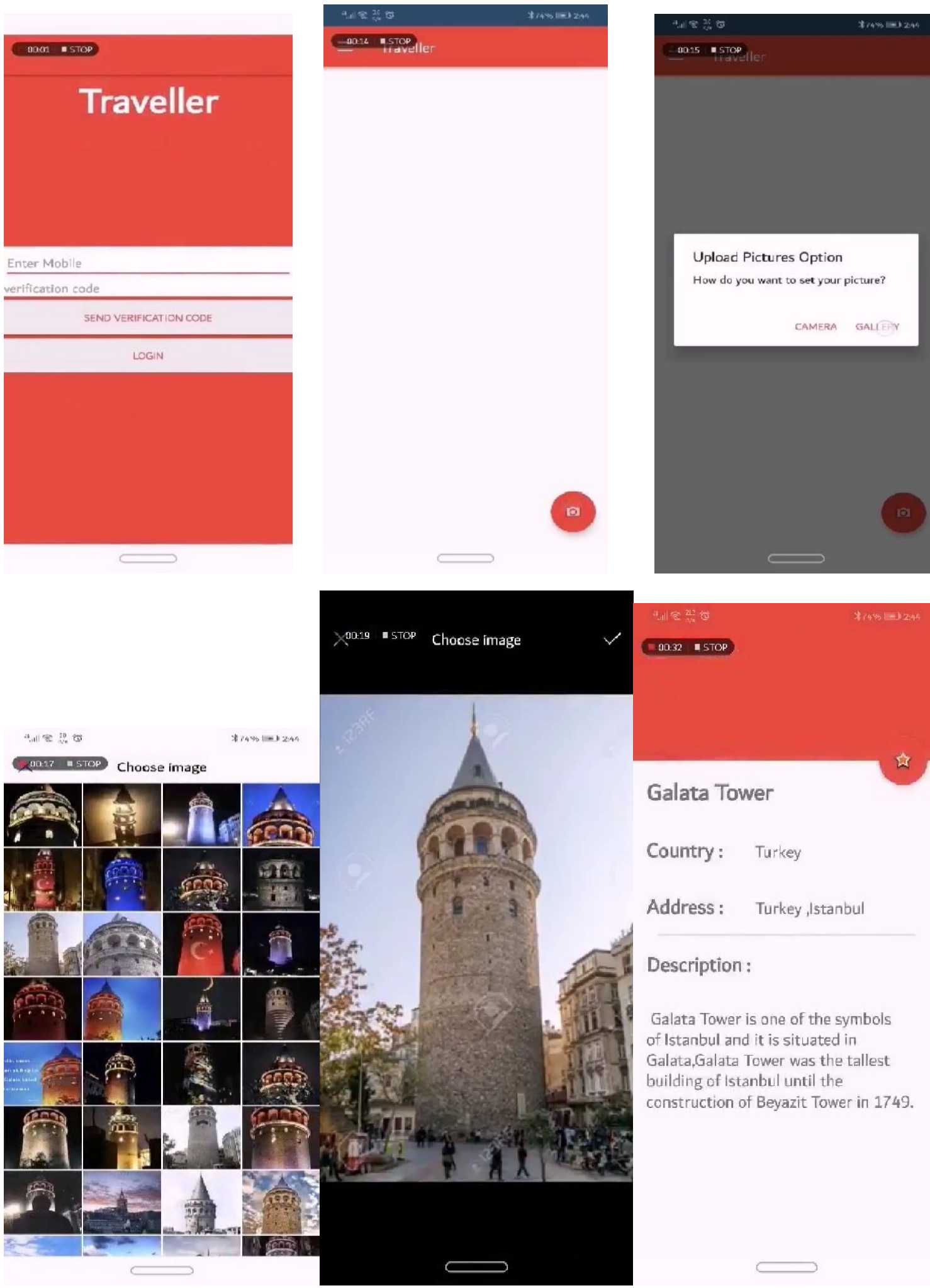

Galata Tower

Country: Turkey

Address : Turkey, lstanbul

Description :

Galata Tower is one of the symbols

of Istanbul and it is situated in

Galata,Galata Tower was the tallest

building of Istanbul until the

construction of Beyazit Tower in 1749. 


\section{4- Conclusion}

It can be stated that the task of architecture classification was performed with decent accuracy for ORB features using descriptor-wise classification with $k$ Nearest Neighbors. Moreover, the latest classification techniques such as multilayer perceptron, recurrent and convolutional neural networks can also be explored for this type of dataset

\section{References :}

[1] https://hadrienj.github.io/posts/Preprocessing-for-deep-learning/

[2] https://www.kaggle.com/egeucak/landmark-places-of-turkey/downloads/landmark-places-ofturkey.zip/1

[3] https://www.researchgate.net/publication/282887055 Fast Image Classificati on for_Monument_Recognition http://slazebni.cs.illinois.edu/publications/ijcv11_landmarks.pdf

[4] http://citeseerx.ist.psu.edu/viewdoc/download?doi=10.1.1.184.8010\&rep=rep1 \&type=pdf

[5] https://pdfs.semanticscholar.org/d68b/5c22161962d78c57685e7c80c99c352ef8bb.pdf?_ga=2.820 $\underline{68246.1286783777 .1544185387-1805921052.1531842828}$

[6] http://ijcsit.com/docs/Volume\%205/vol5issue06/ijcsit20140506139.pdf https://www.researchgate.net/publication/324493420_Image_based_Indian_monument_recogniti on using convoluted neural networks https://pdfs.semanticscholar.org/cc43/a71e05cfc49ab0777b82ca94d18 1f779149f.pdf https://hadrienj.github.io/posts/Preprocessing-for-deep-learning/ https://cvtricks.com/cnn/understand-resnet-alexnet-vgg-inception/ https://towardsdatascience.com/hitchhikers-guide-to-residual-networks -resnet-in-keras385ec01ec8ff http://teleported.in/posts/decoding-resnet-architecture/ 